\title{
Microarray analysis of gene expression in the ovarian cancer cell line HO-8910 with silencing of the ZNF217 gene
}

\author{
GUIQIN SUN $^{1}$, JINGXIA QIN ${ }^{1}$, YUWEN QIU ${ }^{1}$, YUNFEI GAO ${ }^{1}$, \\ YANHONG YU ${ }^{1}$, QINKAI DENG ${ }^{2}$ and MEI ZHONG ${ }^{1}$ \\ ${ }^{1}$ Department of Obstetrics and Gynecology, Nanfang Hospital, Southern Medical University; \\ ${ }^{2}$ Department of Bioinformatics, Southern Medical University, Guangzhou 510515, Guangdong, P.R. China
}

Received April 30, 2009; Accepted July 7, 2009

DOI: 10.3892/mmr_00000183

\begin{abstract}
Zinc-finger protein 217 (ZNF217), which is overexpressed during cancer progression, can promote tumor cell immortalization. To examine the function of ZNF217, a global expression profile was carried out using Affymetrix Gene Chip analysis with HG-U133 plus 2.0 arrays in the ovarian cancer cell line HO-8910 after silencing of the ZNF217 gene. The results were analyzed using the Gene Ontology program to investigate the functional network affected by ZNF217 in ovarian cancer cells. Changes in the mRNA expression of the affected genes were confirmed by real-time reverse transcriptase-polymerase chain reaction (RT-PCR). The results showed that the ZNF217 gene is a key regulator of ovarian cancer, as the silencing of ZNF217 resulted in significant down-regulation by at least 8-fold of 164 genes in the HO-8910 cell line compared to non-silenced control cells. Among these down-regulated genes were ALOX15, CD1D, FXYD3, GAS6, KRT4, LIN7B, MMP-24, PDZK1, PEX6, PRSS8, SLC2A9, STRN and WFDC2. Down-regulation was confirmed by realtime RT-PCR after the silencing of ZNF217 $(\mathrm{p}<0.05)$. The results suggest that $\mathrm{ZNF} 217$ plays a central role in malignant processes in ovarian cancer.
\end{abstract}

\section{Introduction}

Ovarian cancer is among the three most common cancers in Chinese women, and the most common cause of mortality among patients with gynecological tumors. The 5-year survival rate for women presenting with early-stage disease is approximately $90 \%$; however, the majority of women are diagnosed at

Correspondence to: Dr Mei Zhong, Department of Obstetrics and Gynecology, Nanfang Hospital, Southern Medical University, Guangzhou 510515, Guangdong, P.R. China

E-mail: drmzhong@gmail.com

Abbreviations: ZNF217, zinc-finger protein 217

Key words: zinc-finger protein, microarray, ovarian cancer, pathway analysis late stages (stage III or IV) and have a 5-year survival rate of less than $30 \%$ (1). Ovarian cancer is a heterogeneous disease with respect to its histopathology, molecular biology and clinical outcome. Though studies on the etiology of ovarian cancer and susceptibility to the disease have shown that the mechanisms of carcinogenesis and cancer development are associated with genetic, epigenetic and environmental factors, the pathogenesis of ovarian cancer remains unclear (2).

Among the current methods for the identification of gene expression profiles in cancer, microarray analysis is the most powerful. Gene expression profiling using oligonucleotide microarrays is an important source of new insights into the pathogenesis and the initiation/progression mechanisms of cancer. With the advantage of a high-throughput nature, it has been used to identify new candidate oncogenes and different cancer markers, to classify disease entities based on gene expression patterns, and to conduct prognosis or treatment response studies $(3,4)$.

Zinc-finger protein 217 (ZNF217) is thought to be involved in malignant processes in various cancers, such as breast (5), gastric (6), colorectal (7) and prostate (8) cancer. The exact function of ZNF217 in the process of the ovarian cancer is at present unknown. In a previous study, we found that silencing ZNF217 resulted in the effective inhibition of ovarian cancer cell growth and invasiveness (9). Hidaka et al also suggested that ZNF217 was associated with increased metastasis potential in ovarian cancer (7). Additionally, it was reported that the gene was amplified in ovarian carcinoma and might play a central role in the pathogenesis of the disease (10). The present study was designed to determine, using oligonucleotide microarrays, the effect of silencing the ZNF217 gene in HO-8910 cells and the mechanism of ZNF217 in regulating the ovarian cancer signal network. The results suggest that ZNF217 plays a central role in the malignant process in ovarian cancer.

\section{Materials and methods}

Cell line and culture conditions. The human metastasic ovarian cancer cell line HO-8910 was obtained from the ascites of serous cystadenocarcinoma patients. A stable HO-8910/ ZNF217 cell clone was established in our previous study (9). Briefly, shRNA against ZNF127 was inserted into pGensil-1, and the pGensil-1/ZNF217shRNA plasmid was transfected into 
the HO-8910 cells. The cell line was established by selection for 14 days using $1000 \mu \mathrm{g} / \mathrm{ml} \mathrm{G}-418$. HO-8910 cells were cultured in RPMI-1640 medium (Gibco, Grand Island, NY, USA) supplemented with $10 \%$ fetal bovine serum (FBS). HO-8910/ ZNF217- cells were cultured in the above medium with the addition of G-418 $(1000 \mu \mathrm{g} / \mathrm{ml})$. All cultures were grown at $37^{\circ} \mathrm{C}$ in a humidified atmosphere of $95 \%$ air and $5 \%$ carbon dioxide for routine growth. Transfer to fresh medium was performed when confluence reached $90 \%$.

Isolation of RNA. Total RNA was extracted with Trizol reagent (Invitrogen Life Technologies, Carlsbad, CA, USA) from HO-8910 and HO-8910/ZNF217- cells, according to the manufacturer's instructions. The quality of total RNA was determined by the Bioanalyzer (Agilent Technologies, Palo Alto, CA, USA).

Hybridization of RNA to oligonucleotide arrays and data analysis. The Affymetrix Human Genome U133 plus 2.0 Gene Chip oligonucleotide array was used, containing 11 pairs of matched/mismatched 25-mer oligonucleotide probes for each of the 38,500 transcripts of known genes. HO-8910/ZNF217cells were analyzed and compared with HO-8910 cells. For hybridization, $20 \mu \mathrm{g}$ of total RNA per sample was prepared according to the manufacturer's protocol. Fragmented cDNA $(15 \mu \mathrm{g})$ was hybridized to the Human Genome oligonucleotide arrays. The arrays were stained with phycoerythrin-streptavidin, and signal intensity was amplified by treatment with a biotinconjugated antistreptavidin antibody, followed by a second staining using phycoerythrin-streptavidin. After the second staining, the arrays were scanned using the Affymetrix Gene Chip Scanner 3000. Expression data were analyzed using Gene Chip Operating Software 1.1 (Affymetrix) and Gene Spring 6.1 (Silicon Genetics, Redwood City, CA, USA).

Bioinformatics analysis. The Gene Ontology (GO) database (http://www.geneontology.org) was used to annotate and analyze the function of down-regulation genes.

Analysis of mRNA expression by real-time quantitative reverse transcriptase-polymerase chain reaction. Thirteen differentially expressed genes were selected for real-time quantitative reverse transcriptase-polymerase chain reaction (QRT-PCR) analysis with the human $\beta$-actin gene as an internal control. Thus, the reliability of the microarray data was verified by a single method using a Brilliant SYBR Green QPCR Master Mix Kit (Stratagene, La Jolla, CA, USA) and a Mx3000P RealTime PCR System (Stratagene) following the manufacturer's protocol. cDNA was synthesized by oligo dT primed reverse transcription from $2 \mu \mathrm{g}$ of total RNA using an access reverse transcription system (Promega, Madison, WI, USA). Primer sequences are listed in Table I. PCR primers were designed to amplify products of 200-250 bp within target and control sequences. Experiments were performed in triplicate in the same reaction. Target genes and the $\beta$-actin gene were amplified in the same reaction. Comparative quantification was determined using the $2^{-\Delta \Delta \mathrm{Ct}}$ method (11).

Statistical analysis. The Mann-Whitney U test was used to determine the statistical significance of the associations
Table I. Primers used for quantitative reverse transcriptasepolymerase chain reaction.

\begin{tabular}{|c|c|c|}
\hline Gene & Direction & Primer sequence $\left(5^{\prime}-3^{\prime}\right)$ \\
\hline ALOX15 & $\mathrm{F}$ & GCTGGGGCCAAACTATATGA \\
\hline (NM_001140) & $\mathrm{R}$ & CTTGCTCTGACCACACCAGA \\
\hline CD1D & $\mathrm{F}$ & TGAGGCAGCTTTCATCACAC \\
\hline (NM_001766) & $\mathrm{R}$ & GCACACCTTTGCACTTCTGA \\
\hline FXYD3 & $\mathrm{F}$ & TCGCAAGAGGGTCTCTTTGT \\
\hline (NM_005971) & $\mathrm{R}$ & ATATGAGGTCCCATGGCTGA \\
\hline GAS6 & $\mathrm{F}$ & ATCAAGGTCAACAGGGATGC \\
\hline (NM_000820) & $\mathrm{R}$ & CTTCTCCGTTCAGCCAGTTC \\
\hline KRT4 & $\mathrm{F}$ & AGGAGGTCACCATCAACCAG \\
\hline (NM_002272) & $\mathrm{R}$ & GCTCAAGGTTTTTGCTGGAG \\
\hline LIN7B & $\mathrm{F}$ & GCTTTATGACACGCTGGACA \\
\hline (NM_022165) & $\mathrm{R}$ & GATGACCCGGGAGATGTAGA \\
\hline MMP-24 & $\mathrm{F}$ & TGAAGGCATTGACACAGATC \\
\hline (NM_006690) & $\mathrm{R}$ & CGCTCAGTTTCTGGTTGTCA \\
\hline PDZK1 & $\mathrm{F}$ & CTTCAACCCCCGAGAATGTA \\
\hline (NM_002614) & $\mathrm{R}$ & CTTTCAAGTCCACCCGTGTT \\
\hline PEX6 & $\mathrm{F}$ & ATGGCTCCCTTCAGAGGAAT \\
\hline (NM_000287) & $\mathrm{R}$ & TGCAGTTTTGTCTCCACAGC \\
\hline PRSS8 & $\mathrm{F}$ & GTGCCTCTGATCAGTCGTGA \\
\hline (NM_002773) & $\mathrm{R}$ & TTTGGATCCAGGAGGCATAG \\
\hline SLC2A9 & $\mathrm{F}$ & CATCAGCTGGGTCACTGAGA \\
\hline (NM_020041) & $\mathrm{R}$ & TTCACCACCGACAGGTTGTA \\
\hline STRN & $\mathrm{F}$ & CAGATGGCACTCTGCGTTTA \\
\hline (NM_003162) & $\mathrm{R}$ & ATGAGCAGTGATGCTGATCG \\
\hline WFDC2 & $\mathrm{F}$ & CGGCTTCACCCTAGTCTCAG \\
\hline (NM_006103) & $\mathrm{R}$ & AAAGGGAGAAGCTGTGGTCA \\
\hline$\beta$-actin & $\mathrm{F}$ & GATGCAGAAGGAGATCACTG \\
\hline (NM_001101) & $\mathrm{R}$ & GGGTGTAACGCAACTAAGTC \\
\hline
\end{tabular}

F, forward; R, reverse.

between the mRNA expression levels of the HO-8910 and HO-8910/ZNF217- cells. The criterion for statistical significance was $\mathrm{p}<0.05$.

\section{Results}

cDNA microarray analysis. The gene expression profiles of the HO-8910/ZNF217- cells were analyzed using the high-throughput gene chip, which contains 54,675 oligonucleotide-based probe sets. The results of microarray analysis showed that the expression levels of 164 genes were significantly down-regulated by at least 8 -fold in the ovarian cancer cell line with silencing of ZNF217 compared to the non-silenced control cells.

Gene Ontology stat tool for gene expression/microarray analysis. The function of the down-regulated genes was analyzed by the 


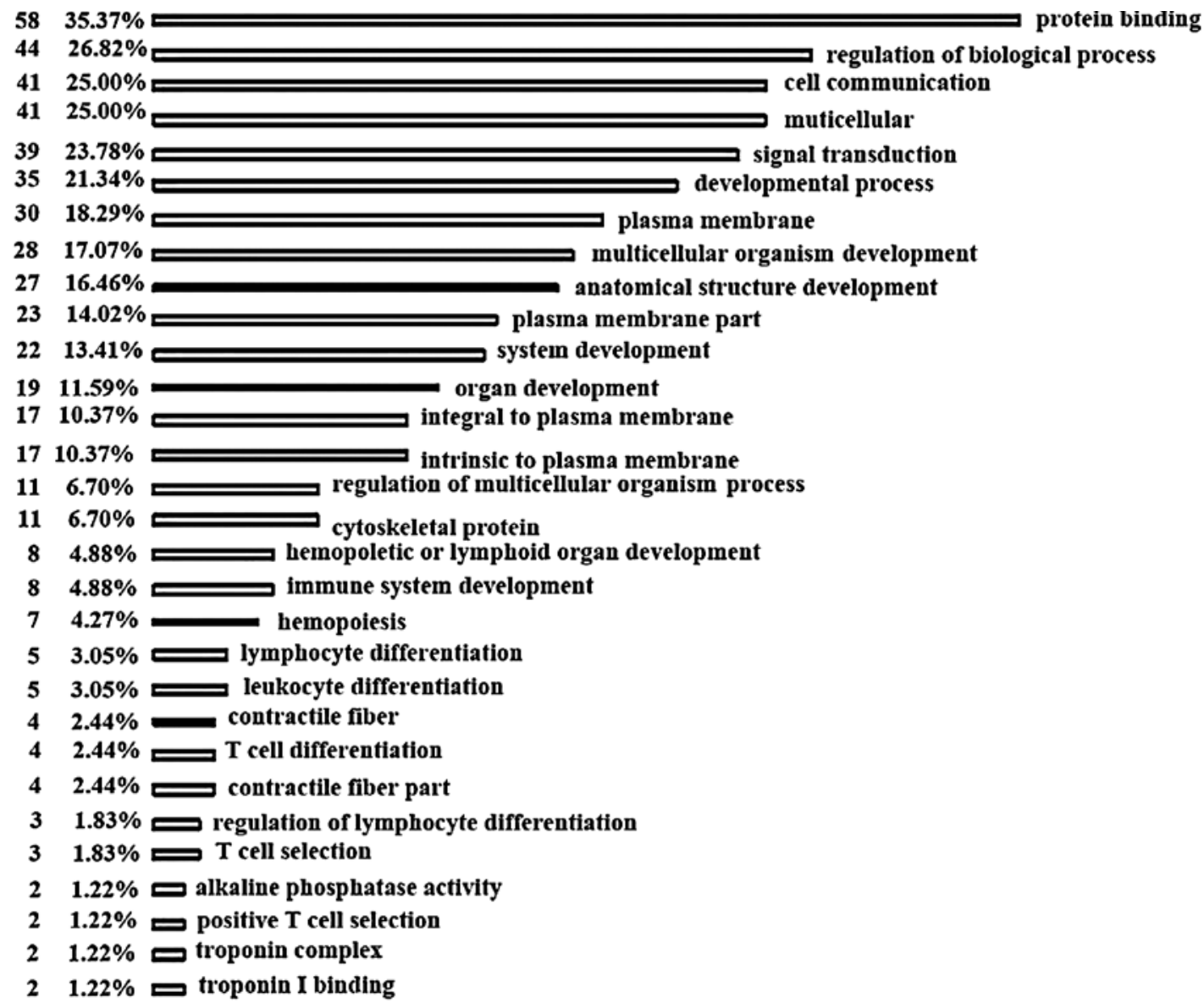

Figure 1. Representation of the distribution of the GO functions of the 164 selected genes. The GO classifications were sorted by percentages. The left column represents the number of genes for specific function. The middle column shows the percentages. The right column shows the name of the different functions. Here, some genes bear multiple functions, which have all been accounted for in their different categories.

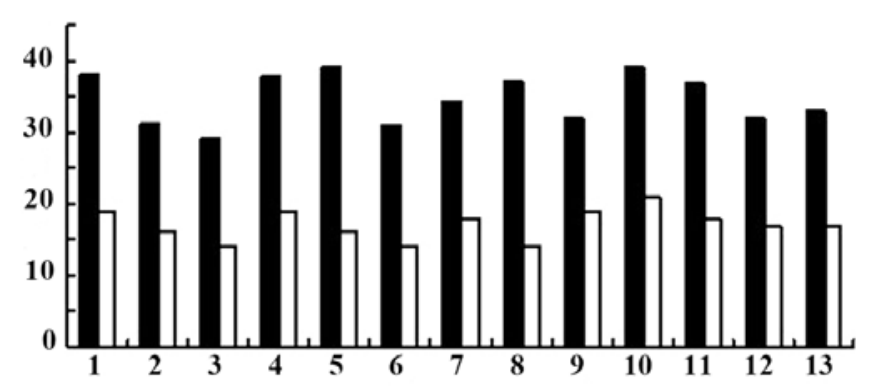

口HO-8910 口HO-8910/ZNF217-

Figure 2. Real-time RT-PCR confirmation of microarray results from a HO-8910/ZNF217- cell line. The graph shows the average fold difference compared with the HO-8910 cell line. The horizontal axis represents the genes analyzed.

GO stat tool. The 164 annotated genes exhibited a wide range of functions (Fig. 1). Genes involved in signal transduction, cell communication, antioxidant activity, cell adhesion, apoptosis and regulation, as well as the cell cycle were targeted for their possible roles in ovarian cancer development.

Validation of microarray data by real-time QRT-PCR analysis. SYBR green-based real-time RT-PCR was used as an inde- pendent method to accurately verify the microarray data. The technique was also used to assess the relative gene expression of a subset of genes that were at least 8-fold down-regulated in the HO-8910/ZNF217- cells. The mRNA expression levels of ALOX15, CD1D, FXYD3, GAS6, KRT4, LIN7B, MMP-24, PDZK1, PEX6, PRSS8, SLC2A9, STRN and WFDC2 as measured by real-time RT-PCR, were highly comparable with the results of microarray analysis (Fig. 2). Down-regulation was confirmed for each gene in HO-8910/ZNF217- cells compared to HO-8910 cells. Although the fold changes observed by real-time RT-PCR were larger than those observed with the microarrays, there was a highly significant correlation between the two methods ( $\mathrm{R}^{2} \sim 0.91$; data not shown).

\section{Discussion}

Ovarian cancer is among the most lethal malignancies worldwide, with a poor prognosis. The molecular mechanisms of the function of ZNF217 in ovarian cancer remain unclear. We therefore sought to assess the function of the protein in ovarian cancer and to identify ZNF217-associated novel marker genes with diagnostic value in ovarian cancer.

Gene expression profiling using high-density microarrays is an excellent tool for the identification, in human cancer, of novel candidate biomarkers associated with the regulation of important cancer-related cellular events, such as cell growth 
and apoptosis. Microarray analysis has great potential for identifying the pathophysiological processes of cancers and carcinomas due to its ability to screen the entire genome and the expression levels of mRNA.

In this study, a stable cell line with silencing of the ZNF217 gene was constructed from the human ovarian cancer cell line HO-8910. Verification tests proved that the ZNF217 gene was down-regulated by $86 \%$ (9). Affymetrix HG-U133 plus 2.0 oligonucleotide microarrays were used to analyze the gene expression pattern in the stable cell line HO-8910/ZNF217and the parent cell line HO-8910. A high-throughput gene chip contain 54,675 oligonucleotide-based probe sets was used to analyze changes in gene expression after the silencing of the ZNF217 gene in HO-8910 cells. Gene expression differed dramatically between the HO-8910/ZNF217- and HO-8910 cells. The 164 genes that were down-regulated by at least 8 -fold were used for the analysis of biological processes and molecular function in ovarian cancer cells.

The Gene Ontology database provides a useful tool for annotating and analyzing the function of large numbers of genes. Modern experimental techniques, e.g., DNA microarrays, often result in long lists of genes. To derive biological information from this kind of data is desirable for functional annotation or the identification of highly represented GO groups. The GO stat program aids in the analysis of such lists, providing statistics related to the GO terms contained in the data and sorting the GO annotations, with the most representative GO terms first.

Compared to the expression profile of the HO-8910 cells, the expression of 164 genes was significantly altered by at least 8 -fold in the HO-8910/ZNF217- cells. Analysis revealed that the functions of these genes were related to signal transduction, cell communication, the regulation of biological processes and protein binding (Fig. 1). According to GO annotation, these genes spanned many members of distinct functional families and biological processes.

Expression levels of the mRNA of thirteen genes selected from the 164 down-regulated genes were verified by real-time RT-PCR. The results show a good correlation between the microarray and RT-PCR data. Among these genes, ALOX15, CD1D, FXYD3, GAS6, KRT4, LIN7B, MMP-24, PDZK1, PEX6, PRSS8, SLC2A9, STRN and WFDC2 were involved in the tumor process.

Of note, the expression of WFDC2 and MMP-24 is closely related to ZNF217 in ovarian cancer cells. Human epididymis4 (HE4), also named WAP four-disulfide core domain 2 (WFDC2), is a member of the Whey Acidic Protein (WAP) family and encodes a WAP-type 4-disulphide core domain-containing protein. The WFDC2 gene is amplified in ovarian carcinomas, whereas its expression in normal tissues, including ovarian tissues, is low $(12,13)$. It has been found to be expressed primarily in certain ovarian cancers, and is thus a marker of ovarian carcinoma (14-16). We found WFDC2 to be downregulated 32-fold after the silencing of the ZNF217 gene. This finding was consistent with that of Zhu et al (17), who reported that WFDC2 and ZNF217 were upregulated through the increased expression of eEF1A2 in lung cancer. Notably, they suggested that eEF1A2 plays an important role in processing. This suggests that eEF1A2 mediates the function of WFDC2 and ZNF217.
Matrix metalloproteinase 24 (MMP-24), also termed MT5-MMP, MMP25 and MT-MMP5, is among the membranetype matrix metalloproteinases (MMPs) on 20q11.2. It is identified and cloned from a human brain cDNA library. The isolated cDNA encodes a polypeptide of 645 amino acids that displays a similar domain organization to that of other MMPs. The MMP-24 gene was down-regulated 32-fold in the human ovarian cancer cell line HO-8910 after silencing of the ZNF217 gene. Members of the MMP family have been well documented as critical players in the breakdown of the extracellular matrix (ECM) under both physiological and pathological conditions. This is important in embryo implantation and cancer progression (18-22). MMP-24 supports the notion that membrane-associated proteolysis plays a critical role in ECM degradation, necessary for many important biological and pathological processes (22-24). It has been reported that MMP-24 expression might have a key function in brain tumor progression (25). Surprisingly, MMP-24 (20q11.2) was frequently co-amplified with ZNF217 (20q13.2) in breast carcinomas (26). A previous study showed that 20q11.2 was amplified in male germ cell tumors, and was related to chemotherapy resistance (27). The present study showed that changes in MMP-24, as an oncogene, are associated with the silencing of the ZNF217 gene. Therefore, ZNF217 may act as a mediator during ovarian cancer progression.

After integrating the information from GO classification and the hierarchical clustering analysis of the selected genes, there appeared to be a series of complicated molecular events associated with ZNF217 activity. Since the information on ZNF217 in ovarian cancer is limited, a comprehensive discussion of all related genes or clusters is impractical. Nevertheless, according to the available information, alterations in the expression of genes involved in anti-oxidation, apoptosis, metastasis, cell adhesion, the cell cycle and signal transduction may highlight the importance of ZNF217 in ovarian cancer cells.

It has been reported that ZNF217 may promote neoplastic transformation and the progression of malignancy (28), and has been associated with tumor progression and poor prognosis in patients with ovarian cancer (29). Our results indicate that ZNF217 is involved in different pathways that may have profound effects on cancer progression (30). The identification of thousands of ZNF217 target genes would enable further studies on the consequences of aberrant expression of ZNF217 during neoplastic transformation (31). Our results suggest that ZNF217 is an ovarian oncogene whose overexpression may break the senesces in normal ovarian epithelium cells, therefore contributing to neoplastic progression. These findings, combined with results from ZNF217-overexpressing lines in ovarian carcinomas, provide a unique model for investigating the interrelationships between these changes and ovarian neoplastic phenotypes (32). Notably, eEF1A2 played a role in mediating ZNF217-induced neoplastic progression (33).

In conclusion, the present study provided a novel and comprehensive expression profile of ovarian cancer cells under the influence of the silenced ZNF217 gene, and identified genes that may be correlated with the process of ovarian cancer. The study shows that ZNF217 in ovarian cancer cells has an effect on multiple intracellular biological events associated with the up-regulation of anti-oxidants, cell growth, apoptosis and cell 
cycle disturbance. Further investigation of the selected genes will provide new insights into the molecular mechanisms of ovarian cancer progression, and will aid in the construction of a stronger ovarian cancer prediction rule.

\section{Acknowledgements}

Grant support was provided by the Guangdong Province Natural Science Foundation of China (No. 8151051501000035). The authors would like to thank Yanqing Ding and Jun Zhou (Department of Pathology, Southern Medical University, Guangzhou, P.R. China) for revision of the manuscript.

\section{References}

1. Fehrmann RS, Li XY, van der Zee AG, et al: Profiling studies in ovarian cancer: a review. Oncologist 12: 960-966, 2007.

2. Aunoble B, Sanches R, Didier E and Bignon YJ: Major oncogenes and tumor suppressor genes involved in epithelial ovarian cancer (Review). Int J Oncol 16: 567-576, 2000.

3. Tothill RW, Kowalczyk A, Rischin D, et al: An expression-based site of origin diagnostic method designed for clinical application to cancer of unknown origin. Cancer Res 65: 4031-4040, 2005.

4. Shipp MA, Ross KN, Tamayo P, et al: Diffuse large B-cell lymphoma outcome prediction by gene-expression profiling and supervised machine learning. Nat Med 8: 68-74, 2002.

5. Nonet GH, Stampfer MR, Chin K, Gray JW, Collins CC and Yaswen P: The ZNF217 gene amplified in breast cancers promotes immortalization of human mammary epithelial cells. Cancer Res 61: 1250-1254, 2001.

6. Zhu YQ, Zhu ZG, Liu BY, Chen XH, Zhang Y and Yin HR: [Study on amplification of ZNF217 in primary gastric carcinoma] Zhonghua Wei Chang Wai Ke Za Zhi 8: 60-62, 2005.

7. Hidaka S, Yasutake T, Takeshita H, et al: Differences in 20q13.2 copy number between colorectal cancers with and without liver metastasis. Clin Cancer Res 6: 2712-2717, 2000.

8. Bar-Shira A, Pinthus JH, Rozovsky U, et al: Multiple genes in human 20q13 chromosomal region are involved in an advanced prostate cancer xenograft. Cancer Res 62: 6803-6807, 2002.

9. Sun G, Zhou J, Yin A, Ding Y and Zhong M: Silencing of ZNF217 gene influences the biological behavior of a human ovarian cancer cell line. Int J Oncol 32: 1065-1071, 2008.

10. Tanner MM, Grenman S, Koul A, et al: Frequent amplification of chromosomal region 20q12-q13 in ovarian cancer. Clin Cancer Res 6: 1833-1839, 2000

11. Livak KJ and Schmittgen TD: Analysis of relative gene expression data using real-time quantitative PCR and the 2(-Delta Delta C(T)) Method. Methods 25: 402-408, 2001.

12. Welsh JB, Zarrinkar PP, Sapinoso LM, et al: Analysis of gene expression profiles in normal and neoplastic ovarian tissue samples identifies candidate molecular markers of epithelial ovarian cancer. Proc Natl Acad Sci USA 98: 1176-1181, 2001.

13. Ono K, Tanaka T, Tsunoda $\mathrm{T}$, et al: Identification by cDNA microarray of genes involved in ovarian carcinogenesis. Cancer Res 60: 5007-5011, 2000.

14. Schummer M, Ng WV, Bumgarner RE, et al: Comparative hybridization of an array of 21,500 ovarian cDNAs for the discovery of genes overexpressed in ovarian carcinomas. Gene 238: 375-385, 1999 .
15. Moore RG, McMeekin DS, Brown AK, et al: A novel multiple marker bioassay utilizing HE4 and CA125 for the prediction of ovarian cancer in patients with a pelvic mass. Gynecol Oncol 112: 40-46, 2009

16. Palmer C, Duan X, Hawley S, et al: Systematic evaluation of candidate blood markers for detecting ovarian cancer. PLoS ONE 3: e2633, 2008

17. Zhu H, Lam DC, Han KC, et al: High resolution analysis of genomic aberrations by metaphase and array comparative genomic hybridization identifies candidate tumour genes in lung cancer cell lines. Cancer Lett 245: 303-314, 2007.

18. Chambers AF and Matrisian LM: Changing views of the role of matrix metalloproteinases in metastasis. J Natl Cancer Inst 89: 1260-1270, 1997.

19. Werb Z, Sympson CJ, Alexander CM, et al: Extracellular matrix remodeling and the regulation of epithelial-stromal interactions during differentiation and involution. Kidney Int Suppl 54: S68-S74, 1996.

20. Werb Z: ECM and cell surface proteolysis: regulating cellular ecology. Cell 91: 439-442, 1997.

21. Woessner JF Jr: Matrix metalloproteinases and their inhibitors in connective tissue remodeling. FASEB J 5: 2145-2154, 1991.

22. Vassalli JD and Pepper MS: Tumour biology. Membrane proteases in focus. Nature 370: 14-15, 1994.

23. Brooks PC, Stromblad S, Sanders LC, et al: Localization of matrix metalloproteinase MMP-2 to the surface of invasive cells by interaction with integrin alpha $\mathrm{v}$ beta 3 . Cell 85 : 683-693, 1996.

24. Nagase H: Cell surface activation of progelatinase A (proMMP-2) and cell migration. Cell Res 8: 179-186, 1998.

25. Llano E, Pendas AM, Freije JP, et al: Identification and characterization of human MT5-MMP, a new membrane-bound activator of progelatinase a overexpressed in brain tumors. Cancer Res 59: 2570-2576, 1999.

26. Tanner MM, Tirkkonen M, Kallioniemi A, et al: Independent amplification and frequent co-amplification of three nonsyntenic regions on the long arm of chromosome 20 in human breast cancer. Cancer Res 56: 3441-3445, 1996.

27. Rao PH, Houldsworth J, Palanisamy N, et al: Chromosomal amplification is associated with cisplatin resistance of human male germ cell tumors. Cancer Res 58: 4260-4263, 1998.

28. Huang G, Krig S, Kowbel D, et al: ZNF217 suppresses cell death associated with chemotherapy and telomere dysfunction. Hum Mol Genet 14: 3219-3225, 2005.

29. Li J, Zhong M, Song LL and Su GD: [Oncogene ZNF217 amplification on chromosome $20 \mathrm{q}$ in ovarian serous cystadenocarcinoma and its clinical implications]. Nan Fang Yi Ke Da Xue Xue Bao 26: 824-825, 2006.

30. Cowger JJ, Zhao Q, Isovic M and Torchia J: Biochemical characterization of the zinc-finger protein 217 transcriptional repressor complex: identification of a ZNF217 consensus recognition sequence. Oncogene 26: 3378-3386, 2007.

31. Krig SR, Jin VX, Bieda MC, et al: Identification of genes directly regulated by the oncogene ZNF217 using chromatin immunoprecipitation (ChIP)-chip assays. J Biol Chem 282: 9703-9712, 2007.

32. Li P, Maines-Bandiera S, Kuo WL, et al: Multiple roles of the candidate oncogene ZNF217 in ovarian epithelial neoplastic progression. Int J Cancer 120: 1863-1873, 2007.

33. Sun Y, Wong N, Guan Y, et al: The eukaryotic translation elongation factor eEF1A2 induces neoplastic properties and mediates tumorigenic effects of ZNF217 in precursor cells of human ovarian carcinomas. Int J Cancer 123: 1761-1769, 2008. 